\title{
Mucosal interleukin-21 mRNA expression level is high in patients with Helicobacter pylori and is associated with the severity of gastritis
}

\author{
NADER BAGHERI ${ }^{1}$, FATEMEH AZADEGAN-DEHKORDI ${ }^{2}$, MAHSA SHIRZAD ${ }^{3}$, \\ BENHAM ZAMANZAD ${ }^{2}$, GHORBANALI RAHIMIAN ${ }^{4}$, AFSHIN TAGHIKHANI', \\ MAHMOUD RAFIEIAN-KOPAEI ${ }^{5}$ HEDAYATOLLAH SHIRZAD ${ }^{2}$
}

${ }^{1}$ Department of Immunology, School of Public Health, Tehran University of Medical Sciences, Tehran, Iran ${ }^{2}$ Cellular and Molecular Research Centre, Shahrekord University of Medical Sciences, Shahrekord, Iran ${ }^{3}$ Faculty of Dentistry, Isfahan University of Medical Sciences, Isfahan, Iran

${ }^{4}$ Department of Internal Medicine, Shahrekord University of Medical Sciences, Shahrekord, Iran

${ }^{5}$ Medical Plants Research Centre, Shahrekord University of Medical Sciences, Shahrekord, Iran

\begin{abstract}
Helicobacter pylori (H. pylori) infection is associated with gastritis and marked infiltration of the gastric mucosa by several cytokines secreting inflammatory cells. Different clinical forms of the infection may reflect distinctive patterns of cytokine expression. Interleukin (IL)-17, IL-21, IL-22, and $I L-23$ have been reported to be involved in $H$. pylori-induced gastric mucosal inflammation, but the details and relationship to different patterns of inflammation and virulence factors remain unclear. The present study was launched to analyse IL-6 expression in H. pylori-infected and uninfected gastric patients and to investigate its correlation with chronic gastritis among $H$. pylori-infected patients. Total RNA was extracted from the gastric antrum biopsies of $48 \mathrm{H}$. pylori-infected patients and $38 \mathrm{H}$. pylori uninfected patients. Mucosal IL-21 mRNA expression level in H. pylori-infected and uninfected gastric biopsy was determined by real-time PCR. The presence of vacA (vacuolating cytotoxin $A)$ and cagA (cytotoxin associated gene A) virulence factors were evaluated using PCR. Interleukin-21 mRNA expression was significantly high in biopsies of $H$. pylori-infected patients compared to $H$. pylori uninfected patients, and the mucosal IL-21 mRNA level was positively correlated with the grade of chronic inflammation. There was no association between virulence factors and IL-21 mRNA expression. We believe that IL-21 might be involved in the pathogenesis of H. pylori and might be an index of the severity of chronic gastritis.
\end{abstract}

Key words: Helicobacter pylori, gastritis, IL-21, virulence factors.

(Centr Eur Immunol 2015; 40 (1): 61-67)

\section{Introduction}

Colonisation by Helicobacter pylori is known to cause chronic gastritis and leads to the development of severe gastroduodenal diseases such as peptic ulcer, lymphoma of the mucosa associated lymphoid tissue (MALT), or gastric adenocarcinoma [1]. Helicobacter pylori infection is associated with gastritis and considerable infiltration of neutrophils, monocytes, and lymphocytes into the gastric mucosa, which contribute to maintaining and expanding local inflammation. Activation and migration of these in- flammatory cells into the gastric mucosa is related to increased production of pro-inflammatory cytokines, which are believed to contribute in maintaining the gastric inflammation and causing epithelial cell damage [2-4]. The risk of different clinical expression of $H$. pylori infection is thought to rely on interaction between the host genetic factors and bacterial factors. For instance, polymorphisms of pro-inflammatory cytokine genes such as interleukin (IL)-8, IL-10, IL-17, IL-1 $\alpha$, and tumor necrosis factor $\alpha$ $(\mathrm{TNF}-\alpha)$, which enhance inflammatory response of gastric mucosa and have been correlated to an increased risk of

Correspondence: Prof. Hedayatollah Shirzad, Cellular and Molecular Research Centre, Shahrekord University of Medical Sciences, phone +98-9131859510, fax +98-3813330709, e-mail: shirzad1951@yahoo.com or shirzadeh@SKUMS.ac.ir 
gastric cancer and peptic ulcer [5, 6]. It has been shown that the mucosal levels of several cytokines were significantly higher in $H$. pylori-infected patients with virulence factors in comparison to $H$. pylori-infected patients without virulence factors $[7,8]$. It is suggested that the clinical outcome of $H$. pylori infection is linked to certain strains such as the cytotoxin-associated gene (cagA) and vacuolating cytotoxin (vacA) [9]. The $\operatorname{cagA}$ gene, which is a marker for the presence of a pathogenicity island (cagPAI), has been shown to be involved in induction of pro-inflammatory chemokine release, which is also associated with more severe gastritis and higher prevalence of peptic ulcer and gastric cancer [10-12]. Vacuolating cytotoxin A has two variable parts: the $\mathrm{s}$ (signal peptide) region exists as allele $\mathrm{s} 1$ or $\mathrm{s} 2$; and the $\mathrm{m}$ (middle) region occurs as $\mathrm{m} 1$ or $\mathrm{m} 2$ allelic types. The allele combination of $\mathrm{s} 1 \mathrm{~m} 1$ confers high, the $\mathrm{s} 1 \mathrm{~m} 2$ intermediate, and the $\mathrm{s} 2 \mathrm{~m} 2$ low toxin activity. The vacA s1 strain is mostly cagA-positive, thus the two markers are closely related [13]. There are geographic differences between vacA status and $H$. pylori-related diseases. In Western countries infection with vacA s1 strain is more common in patients with peptic ulcer than in those with chronic gastritis. However, in Asian populations the association between vacA diversity and clinical outcome is not established [14]. Interleukin-21 (IL-21) is a four-helix bundle cytokine that is produced by activated $\mathrm{CD}^{+}$ $\mathrm{T}$ cells and natural killer $\mathrm{T}$ (NKT) cells $[15,16]$. T follicular helper (Tfh) cells also produce IL-21, which regulates generation of humoral responses and germinal centre formation [17]. Interleukin-21 can stimulate non-immune cells to synthesise various inammatory molecules [15]. Helper $\mathrm{T}$ cells that produce IL-17 ('TH-17 cells') are distinct subsets of pro-inflammatory cells whose in vivo function requires IL-23 but whose in vitro differentiation requires only IL-6 and transforming growth factor- $\beta 1$ (TGF- $\beta 1$ ). Studies have demonstrated that IL-6 induces expression of IL-21 that amplifies an autocrine loop to induce more IL-21 and IL-23 receptors in naive CD4 ${ }^{+} \mathrm{T}$ cells. Both IL-23 and IL-21, along with TGF- $\beta 1$, induce IL-17 expression independently of IL-6. Interleukin-21 and IL-23 induce the orphan nuclear receptor $\mathrm{ROR} \gamma \mathrm{t}$, which in synergy with STAT3 enhances IL-17 expression. 'TH-17' cells produce IL-17, IL-17F, IL-21, and IL-22, which are thought to be required for the control of a variety of bacterial and fungal infections at mucosal surfaces $[18,19]$. High levels of IL-21 expression occur in human Th1-associated diseases, and administration of IL-21 to mice enhances the inux of immune cells into inamed tissues and the severity of immune-mediated diseases $[20,21]$. In the light of this study, it is conceivable that IL-21 may play an important role in the initiation and perpetuation of chronic infiammatory processes and may be associated with virulence factors. We have therefore sought to determine the role of IL-21 in $H$. pylori-associated gastritis. We firstly examined IL-21 in the gastric mucosa of patients with
H. pylori infection. Secondly, we evaluated the effect of virulence factors cagA and vacA allelic variants in $H$. $p y$ lori-infected on the mucosal IL-21 mRNA level in gastric mucosa. Finally, we determined the correlation between mucosal IL-21 mRNA levels and grade of gastritis.

\section{Material and methods}

\section{Patients and sampling}

A total of 86 subjects undergoing endoscopy at Hajar University Hospital, in Shahrekord, Iran, were included in the study. The study was approved by the Human Research Ethics Committee of Shahrekord University of Medical Sciences, and informed consent was obtained from each volunteer before participation. The groups were characterised as follows: the $H$. pylori-positive group $(n=48$ : 16 males, 32 females; mean age: $42.4 \pm 14.82$ years); the H. pylori-negative group ( $n=38: 18$ males, 20 females; mean age: $38.18 \pm 16.51$ years). Helicobacter pylori infection was determined by the rapid urease test, PCR (16s rRNA and glmM) and histological examination of biopsies taken from the antrum. Patients were classified as $H$. pylori infected only if the four tests were positive. Four biopsies were collected from $H$. pylori-infected patients, and used for rapid urease test, histological examination, assessment of bacterial virulence factors, detection of $H$. pylori, and cytokine RNA analysis. This study was approved by the Ethics Committee of Shahrekord University of Medical Sciences, Shahrekord, Iran.

\section{Histological examination}

Sections of biopsy specimens were embedded in $10 \%$ buffered formalin and stained with Haematoxylin and Eosin to examine gastritis, and with Giemsa to detect $H$. pylo$r i$. The histological severity of gastritis (acute and chronic) was blindly graded from normal to severe based on the degree of mononuclear cell (MNC) and polymorphonuclear leukocyte (PMN) infiltration according to the Updated Sydney system [22] on a four-point scale: 0 , no; 1 , mild; 2 , moderate; and 3 , severe changes. The grade of chronic and active inflammation was investigated for involvement according to the density of neutrophils and mononuclear in gastric mucosal crypts, from one to all crypts.

\section{Polymerase chain reaction amplification}

DNA for polymerase chain reaction (PCR) was extracted using the Biospin Tissue Genomic DNA Extraction Kit (BioFlux, Japan). Specific primers for PCR amplification of different genes are shown in Table 1. For vacA and cagA evaluation, the PCR program comprised 35 cycles of denaturation (at $94^{\circ} \mathrm{C}$ for $30 \mathrm{~s}$ ), annealing (at $56^{\circ} \mathrm{C}$ for $30 \mathrm{~s}$, extension at $72^{\circ} \mathrm{C}$ for $30 \mathrm{~s}$ ), and one final extension (at $72^{\circ} \mathrm{C}$ for $5 \mathrm{~min}$ ). 
Table 1. Polymerase chain reaction primers for amplification of virulence factors

\begin{tabular}{ccc}
\hline Size of PCR product $(\mathbf{b p})$ & Primer sequence & Primer designation \\
\hline $567 \mathrm{bp}(\mathrm{m} 1)$ & vacAmF: 5-CAATCTGTCCAATCAAGCGAG-3 & vacA m1/m2 \\
$642 \mathrm{bp}(\mathrm{m} 2)$ & vacAmR: 5-GCGTCTAAATAATTCCAAGG-3 & vacA s1/s2 \\
\hline $259 \mathrm{bp}(\mathrm{s} 1)$ & VA1-F: 5-ATGGAAATACAACAAACACAC-3 & cagA \\
\hline $286 \mathrm{bp}(\mathrm{s} 2)$ & VA1-R: 5-CTGCTTGAATGCGCCAAAC-3 & \\
\hline $232 \mathrm{bp}$ & cag1: 5-ATGACTAACGAAACTATTGATC-3 & \\
\hline
\end{tabular}

\section{Quantitative analysis for interleukin-21 mRNA in the gastric mucosa using real-time polymerase chain reaction}

Total RNA was isolated from whole gastric biopsy specimens using total RNA extraction biozol (Bioflux, Japan). An aliquot containing $0.2 \mu \mathrm{g}$ of total RNA was used for the reverse transcription reaction, which was conducted using the superscript first-strand cDNA synthesis system (Fermentas, Finland) according to the manufacturer's instructions. The sequences of oligonucleotide primer and probe are shown in Table 2. The quantification of IL-21 mRNA levels was performed using a Rotor-Gene 3000 (Corbett). Real-time PCR reactions were performed in a total volume of $25 \mu \mathrm{l}$, containing $3 \mu \mathrm{l}$ of synthesised cDNA solution, $12.5 \mu$ l of $2 x$ Rotor-Gene Probe PCR Master Mix (Qiagen, Germany), $500 \mathrm{nM}$ of each primer, and $250 \mathrm{nM}$ of the TaqMan probe. Amplification program included a pre-warming step $\left(10 \mathrm{~min}\right.$ at $\left.94^{\circ} \mathrm{C}\right)$, denaturation step $\left(94^{\circ} \mathrm{C}\right.$ for $\left.15 \mathrm{~s}\right)$, and an annealing/extension step $\left(60^{\circ} \mathrm{C}\right.$ for $\left.60 \mathrm{~s}\right)$. The expression levels of cytokine mRNA were expressed as the ratio of cytokine mRNA to $\beta$-actin mRNA (cytokine mRNA/ $\beta$-actin mRNA). Each assay was performed in duplicate, and each cytokine assay was performed testing all RNA samples in the same experiment. Relative quantification of cytokine to $\beta$-actin (cytokine mRNA/ $\beta$-actin mRNA) was determined using the $2^{-\Delta \mathrm{Ct}}=$ $=2^{-(\mathrm{Ct}, \mathrm{IL}-21-\mathrm{Ct}, \beta \text {-actin })}$ method.

\section{Statistical analysis}

Data were analysed using GraphPad Prism 5 Demo (GraphPad Software, San Diego, California, USA). Data are expressed as raw and mean if not stated otherwise. Age was analysed by unpaired Student's $t$-test. In statistics, normality tests were used to determine whether a data set was well modelled by a normal distribution or not, cytokine expression was presented as mean, and differences between infected and uninfected groups were analysed using Student's $t$-test. Also for gene expression values between $H$. pylori-infected patients and virulence factors the parametric Student's $t$-test was used, and for comparison of more than two groups one-way ANOVA test was used. Categorical data (between virulence factors) are presented as frequencies; comparisons were made using the $\chi^{2}$ or
Table 2. Primer and probe sequences employed in this study

\begin{tabular}{cc}
\hline Gene & Primer and probe sequence \\
\hline$\beta$-actin & Forward 5-AGCCTCGCCTTTGCCGA-3 \\
& Reverse 5-CTGGTGCCTGGGGCG-3 \\
& Probe FAM-CCGCCGCCCG \\
& TCCACACCCGCC-TAMRA \\
\hline IL-21 & Forward 5- TGTGAATGACTTGGTCCCTGAA-3 \\
& Reverse 5- AACAGGAAAAAGCTGACCACTCA-3 \\
& Probe FAM-TCTGCCAGCTCCAGAAGATG \\
& TAGAGACAAAC-TAMRA \\
\hline
\end{tabular}

Fisher exact test if $<5$ samples were in one group. $P$ values $<0.05$ were considered to be statistically significant.

\section{Results}

\section{Correlation of vacA alleles and presence of cagA gene}

The correlation of $\mathrm{s} 1$ and $\mathrm{s} 1 \mathrm{~m} 2$ genotypes with cagA positive was statistically significant, and no correlation of $\mathrm{m} 1$ genotype was observed with cagA. The cagA gene was detected in $60.4 \%$ (29 strains) of $H$. pylori. The association between vacA alleles and the presence of virulence factors

Table 3. Correlation of vacA alleles with the cagA genotype of the samples studied

\begin{tabular}{|c|c|c|}
\hline vacA genotype & $\begin{array}{c}\text { cagA } \\
\text { Positive }\end{array}$ & Negative \\
\hline $\mathrm{s} 1 \mathrm{~m} 1$ & 7 & 4 \\
\hline $\mathrm{s} 1 \mathrm{~m} 2$ & 16 & 6 \\
\hline $\mathrm{s} 2 \mathrm{~m} 2$ & 2 & 6 \\
\hline$P$ value & \multicolumn{2}{|c|}{0.06} \\
\hline s1 & 26 & 11 \\
\hline s2 & 2 & 6 \\
\hline$P$ value & \multicolumn{2}{|c|}{0.018} \\
\hline $\mathrm{m} 1$ & 7 & 4 \\
\hline $\mathrm{m} 2$ & 22 & 14 \\
\hline$P$ value & \multicolumn{2}{|c|}{0.881} \\
\hline
\end{tabular}




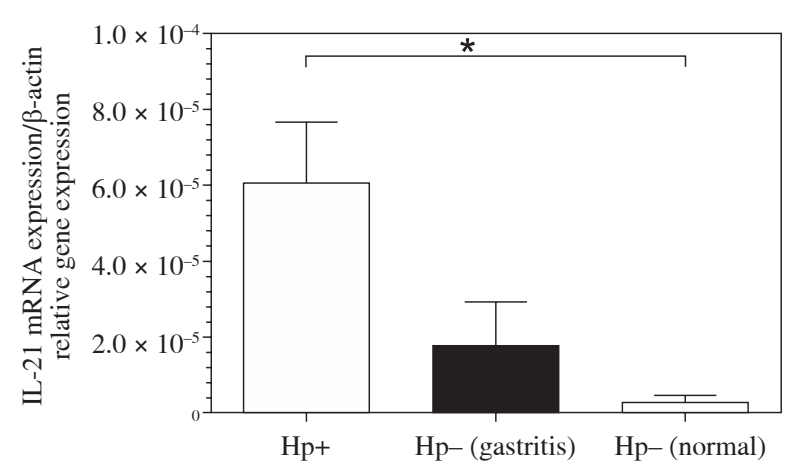

Fig. 1. Relative expression level of interleukin (IL)-21 mRNA in gastric mucosa shown by Helicobacter pylori status. RNA was extracted from gastric biopsies of $48 \mathrm{H}$. pylori-infected patients, $26 \mathrm{H}$. pylori-negative patients with gastritis, $12 \mathrm{H}$. pylori-negative normal mucosa; and analysed for IL-21 by real-time polymerase chain reaction. Levels are normalised to $\beta$-actin. Statistical significance was evaluated using the one-way ANOVA test $(* p<0.05)$

is described in Table 3. Out of 29 H. pylori subjects that were positive for cagA, 26 strains were associated with the toxin-producing vacA s1, and only 2 cagA-positive strains were vacA s2 $(p<0.018)$. Sixteen strains were associated with the toxin-producing vacA s1m2, only 7 strains were vacA s $1 \mathrm{~m} 2$, and 2 strains were vacA s $2 \mathrm{~m} 2(p<0.06)$.

\section{Interleukin-21 expression in Helicobacter pylori- infected gastric mucosa}

Interleukin-21 expression was significantly greater in biopsy specimens of $H$. pylori infected patients compared with $H$. pylori negative (Fig. 1). If the mean in the H. pylori-positive group is 0.00006 and the mean in the

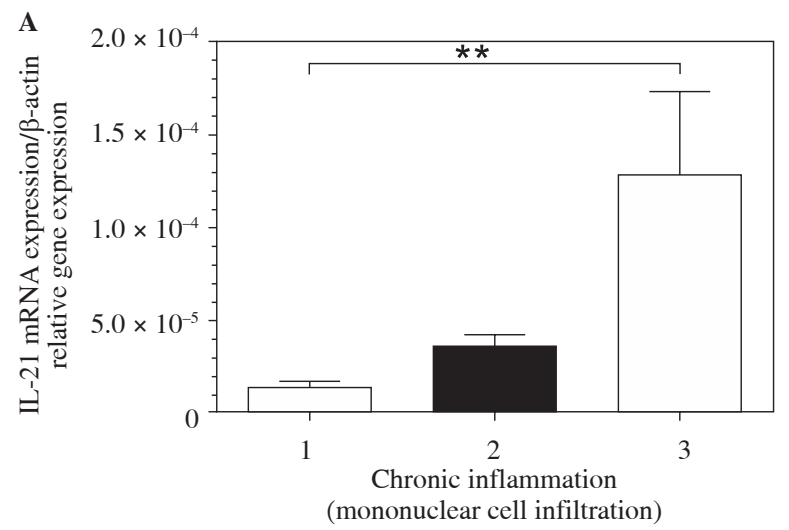

H. pylori-negative patients with gastritis is 0.000017 , then the difference in the IL-21 mRNA expression in the $H$. pylori-positive patients compared with the $H$. pylori-negative patients with gastritis is $0.00006 / 0.000017$, or 3.5 fold. Also, the difference in the IL-21 mRNA expression in the $H$. pylori-positive patients compared with the $H$. $p y$ lori-negative normal mucosa is $0.00006 / 0.0000027$, or 22.22 fold, and $H$. pylori-negative patients with gastritis compared with the $H$. pylori-negative normal mucosa is $0.000017 / 0.0000027$, or 6.3 fold.

\section{The effect of vacA allelic variants in Helicobacter pylori infected on the mucosal interleukin-21 mRNA level in gastric mucosa}

Our results showed that in $H$. pylori-infected patients, the mucosal IL-21 mRNA level was independent of the vacA status. The difference in the IL-21 mRNA expression in the vacA s1-positive $H$. pylori strains compared with the vacA s2-positive $H$. pylori strains was $0.00008 / 0.000041$, or 1.95 fold. Mucosal IL-21 mRNA expression in H. pylori-infected patients with vacA ml-positive was not significantly higher than those observed in $H$. pylori-infected patients with vacA m2-positive (0.00008/0.00007, or 1.19 fold). Also, mucosal IL-21 mRNA expression in gastritis patients with vacA s1ml-positive was not significantly higher than those observed in gastritis patients with vacA s1m2-positive (0.000084/0.000089, or 0.94 fold) and vacA s $2 \mathrm{~m} 2$-positive (0.000084/0.000041, or 2.05 fold).

\section{The effect of cagA virulence factor in Helicobacter pylori infected on the mucosal interleukin-21 mRNA level in gastric mucosa}

Mucosal IL-21 mRNA level was independent of cagA virulence factor status. If the mean $H$. pylori-positive

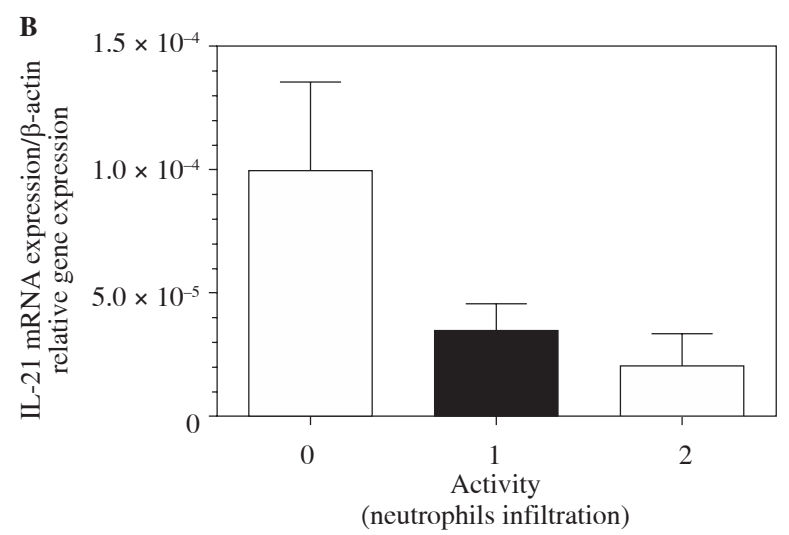

Fig. 2. Relative expression levels of interleukin (IL)-21 mRNA in Helicobacter pylori-infected patients shown by grade of gastritis. The mucosal IL-21 mRNA level is positively correlated with the grade of chronic inflammation (A). Also, no correlation was seen between the mucosal IL-21 mRNA expression and the activity score (B). Statistical significance was evaluated using Student's $t$-test $(* * p<0.001 ; p>0.99)$ 
group with cagA is 0.00007 and the mean $H$. pylori-positive group without cagA is 0.00003 , then the difference in the IL-21 mRNA expression in the cagA-positive $H$. pylori strains compared with the cagA-negative H. pylori strains is $0.00007 / 0.00003$, or 2.3 fold.

\section{Correlation between mucosal interleukin-21 mRNA levels and grade of gastritis}

The degree of inflammatory activity was assessed and graded as follows: $54.1 \%$ (26) none, $39.6 \%$ (19) mild, and $6.3 \%$ (3) moderate. As far as chronic inflammation was concerned, there were $37.5 \%$ (18) mild, $33.3 \%$ (16) moderate, and $29.1 \%$ (14) severe in the studied population. Figure 2 shows the relationship between the mucosal IL-21 mRNA expression and the activity (neutrophil infiltration) and chronic inflammation (mononuclear cell infiltration) scores. There was a significant correlation between the mucosal IL-21 mRNA expression and the chronic inflammation scores. No correlation was observed between the mucosal IL-21 mRNA expression and the activity score.

\section{Discussion}

Our findings showed that the mucosal IL-21 mRNA level increased in the $H$. pylori-infected patients compared with uninfected patients and correlated positively with the degree of chronic inflammation in patients infected with $H$. pylori. Mucosal IL-21 mRNA expression was also independent of the virulence factors of $H$. pylori. Similarly to other reports [23], we also found that the correlation of s1 and $\mathrm{s} 1 \mathrm{~m} 2$ genotypes with cagA-positive was statistically significant, but no correlation between $\mathrm{m} 1$ genotype and cagA was observed. We concluded that the dominant genotypes of $H$. pylori in Iranian patients with chronic gastritis might be cagA-positive s1/m2. Interleukin-21 is over-produced in other Th1-associated infiammatory diseases of the gastro-intestinal tract, such as $H$. pylori-related gastritis and celiac disease [24, 25]. The results of Caruso et al. indicate that IL-21 was over expressed in $H$. pylori-infected gastric mucosa and gastric epithelial AGS cells response to IL-21, increasing production of matrix metalloproteinase 2 (MMP-2) and MMP-9, (but not MMP-1, MMP-3, or MMP-7) which contribute to mucosal ulceration and epithelial damage [24]. High IL-21 production was also documented in autoimmune diseases such as psoriasis [26], atopic dermatitis (AD) [27], systemic lupus erythematosus (SLE) [28], graft-versus-host disease (GVHD) [29], and multiple sclerosis [30]. The data emerging from these studies show that IL-21 has broad effects on both innate and adaptive immune responses, and excessive production of IL-21 associates with destructive immune reactions in various organs. However, the basic mechanism underlying IL-21 synthesis in diseased organs remains to be clarified. The study by Sutherland et al. demonstrated that IL-21 levels were increased in the pancreas as NOD mice de- veloped diabetes, and that $\mathrm{CD}^{+}{ }^{+}$and $\mathrm{CD} 8^{+} \mathrm{T}$-cells infiltrating the pancreas could respond to local IL-21 as they expressed IL-21R [31]. These data are consistent with recent studies from the laboratories of Leonard and Sarvetnick [32], showing that IL-21 $\mathrm{R}^{-/}$NOD mice are protected from insulitis and type 1 diabetes. Interleukin- 21 could also play a role in the pathogenesis of other immune-mediated pathologies as its levels were found to be up-regulated in synovial tissue samples of patients with rheumatoid arthritis (RA) and in labial salivary gland sections of patients with Sjögren's syndrome (SS), as well as in the plasma of patients with SS, patients with Behçet disease, and patients with Hashimoto's thyroiditis [33]. A previous published study showed that IL-21 was not able to trigger Th1 cell differentiation, but it could enhance ongoing Th1 cell responses. For example, Strengell et al. indicated that IL-21 enhanced interferon $\gamma$ (IFN- $\gamma$ ) gene expression in activated human $\mathrm{T}$ cells and promoted the transcription of Th1-associated genes, including T-bet [34]. The same group also documented a synergistic effect of IL-21 and IL-15 or IL- 21 and IL-18 on IFN- $\gamma$ mRNA synthesis and production [35], whereas Kasaian et al. demonstrated that IL-21 cooperates with IL- 2 and IL- 15 in promoting IFN- $\gamma$ synthesis [36]. Finally, a study previously showed that IL-21 enhanced T-bet and p-Stat4 expression and sustained IFN- $\gamma$ synthesis in gut mucosal lymphocytes of patients with Crohn's disease [20]. A recent study indicated an increase in Th17 in $H$. pylori-infected patients and in human gastric tumours, and further showed that $H$. pylori-primed GMF (gastric myofibroblasts/fibroblasts) promoted differentiation of TH-17. This process was dependent on IL-6, TGF- $\beta 1$, and IL-21, which $H$. pylori-exposed and gastric tumour-derived MF (myofibroblast and fibroblast) produced at increased levels, consequently maintaining a stronger ability to induce TH-17 cells. Their findings suggest that the enhanced $\mathrm{TH}-17$ promoting capacity of the GMF, derived from gastric tumours, might be among the key factors contributing to gastric tumour-promoting inflammatory milieu [37]. Another study indicated that IL-21 expression was markedly higher in human gastric cancer mucosa compared to normal [38]. Interleukin-21 can maintain chronic inflammation and/or favour tissue damage by promoting the recruitment of immune cells within the inflamed tissue [39], the expansion of autoreactive T cells [40], and the synthesis of extracellular matrix metalloproteinases [24].

The authors declare no conflict of interest.

This study was derived from the MSc thesis of the first author and was financially supported by the research deputy of Shahrekord University of Medial Sciences. The authors are grateful to the staff of the Cellular \& Molecular Research Centre, Shahrekord University of Medical Sciences, and the authorities of the endoscopy unit of Shahrekord Hajar Hospital for their valuable help. 


\section{References}

1. Salimzadeh L, Bagheri N, Zamanzad B, et al. (2015) Frequency of virulence factors in Helicobacter pylori-infected patients with gastritis, Microb Pathog 80: 67-72.

2. Bagheri N, Azadegan-Dehkordi F, Shirzad H, et al. (2015) The biological functions of IL-17 in different clinical expressions of Helicobacter pylori-infection. Microb Pathog 81: 33-8.

3. Bagheri N, Taghikhani A, Rahimian G, et al. (2013): Association between virulence factors of helicobacter pylori and gastric mucosal interleukin-18 mRNA expression in dyspeptic patients. Microb Pathog 65: 7-13.

4. Rahimian G, Sanei MH, Shirzad H, et al. (2014): Virulence factors of Helicobacter pylori vacA increase markedly gastric mucosal TGF- $\beta 1$ mRNA expression in gastritis patients. Microb Pathog 67-68: 1-7.

5. Sugimoto M, Furuta T, Shirai N, et al. (2007): Different effects of polymorphisms of tumor necrosis factor-alpha and interleukin-1 beta on development of peptic ulcer and gastric cancer. J Gastroenterol Hepatol 22: 51-59.

6. Yuzhalin A (2011): The role of interleukin DNA polymorphisms in gastric cancer. Hum Immunol 72: 1128-1136.

7. Yamaoka Y, Kita M, Kodama T, et al. (1997): Induction of various cytokines and development of severe mucosal inflammation by cagA gene positive Helicobacter pylori strains. Gut 41: 442-451.

8. Bagheri N, Rahimian G, Salimzadeh L, et al. (2013): Association of the virulence factors of Helicobacter pylori and gastric mucosal interleukin-17/23 mRNA expression in dyspeptic patients. EXCLI J 12: 5-14

9. Basso D, Plebani M (2004): H. pylori infection: bacterial virulence factors and cytokine gene polymorphisms as determinants of infection outcome. Crit Rev Clin Lab Sci 41: 313-337.

10. Brandt S, Kwok T, Hartig R, et al. (2005): NF-kappaB activation and potentiation of proinflammatory responses by the Helicobacter pylori CagA protein. Proc Natl Acad Sci U S A 102: 9300-9305.

11. Yamaoka Y, Souchek J, Odenbreit S, et al. (2002): Discrimination between cases of duodenal ulcer and gastritis on the basis of putative virulence factors of Helicobacter pylori. J Clin Microbiol 40: 2244-2246.

12. Bagheri N, Azadegan-Dehkordi F, Sanei H, et al. (2014): Associations of a TLR4 single-nucleotide polymorphism with H. pylori associated gastric diseases in Iranian patients. Clin Res Hepatol Gastroenterol 38: 366-371.

13. Atherton JC, Cao P, Peek RM Jr, et al. (1995): Mosaicism in vacuolating cytotoxin alleles of Helicobacter pylori. Association of specific vacA types with cytotoxin production and peptic ulceration. J Biol Chem 270: 17771-17777.

14. Yamaoka Y, Kodama T, Kita M, et al. (1998): Relationship of vacA genotypes of Helicobacter pylori to cagA status, cytotoxin production, and clinical outcome. Helicobacter 3: 241-253.

15. Parrish-Novak J, Dillon SR, Nelson A, et al. (2000): Interleukin 21 and its receptor are involved in NK cell expansion and regulation of lymphocyte function. Nature 408: 57-63.

16. Coquet JM, Kyparissoudis K, Pellicci DG, et al. (2007): IL21 is produced by NKT cells and modulates NKT cell activation and cytokine production. J Immunol 178: 2827-2834.

17. Spolski R, Leonard WJ (2008): Interleukin-21: basic biology and implications for cancer and autoimmunity. Annu Rev Immunol 26: 57-79.

18. Zhou L, Ivanov II, Spolski R, et al. (2007): IL-6 programs $\mathrm{T}(\mathrm{H})-17$ cell differentiation by promoting sequential engage- ment of the IL-21 and IL-23 pathways. Nat Immunol 8: 967974.

19. Liang SC, Tan XY, Luxenberg DP, et al. (2006): Interleukin (IL)-22 and IL-17 are coexpressed by Th17 cells and cooperatively enhance expression of antimicrobial peptides. J Exp Med 203: 2271-2279.

20. Monteleone G, Monteleone I, Fina D, et al. (2005): Interleukin-21 enhances T-helper cell type I signaling and interferon-gamma production in Crohn's disease. Gastroenterology 128: 687-694.

21. Vollmer TL, Liu R, Price M, et al. (2005): Differential effects of IL-21 during initiation and progression of autoimmunity against neuroantigen. J Immunol 174: 2696-2701.

22. Manxhuka-Kerliu S, Telaku S, Devolli-Disha E, et al. (2009): Helicobacter pylori gastritis updated Sydney classification applied in our material. Prilozi 30: 45-60.

23. Warburton VJ, Everett S, Mapstone NP, et al. (1998): Clinical and histological associations of cagA and vacA genotypes in Helicobacter pylori gastritis. J Clin Pathol 51: 55-61.

24. Caruso R, Fina D, Peluso I, et al. (2007): IL-21 is highly produced in Helicobacter pylori-infected gastric mucosa and promotes gelatinases synthesis. J Immunol 178: 5957-5965.

25. Fina D, Sarra M, Caruso R, et al. (2008): Interleukin 21 contributes to the mucosal $\mathrm{T}$ helper cell type 1 response in coeliac disease. Gut 57: 887-892.

26. Caruso R, Botti E, Sarra M, et al. (2009): Involvement of interleukin-21 in the epidermal hyperplasia of psoriasis. Nat Med 15: 1013-1015.

27. Jin H, Oyoshi MK, Le Y, et al. (2009): IL-21R is essential for epicutaneous sensitization and allergic skin inflammation in humans and mice. J Clin Invest 119: 47-60.

28. Wong CK, Wong PT, Tam LS, et al. (2010): Elevated production of B cell chemokine CXCL13 is correlated with systemic lupus erythematosus disease activity. J Clin Immunol 30: 45-52.

29. Hippen KL, Bucher C, Schirm DK, et al. (2012): Blocking IL-21 signaling ameliorates xenogeneic GVHD induced by human lymphocytes. Blood 119: 619-628.

30. Tzartos JS, Craner MJ, Friese MA, et al. (2011): IL-21 and IL-21 receptor expression in lymphocytes and neurons in multiple sclerosis brain. Am J Pathol 178: 794-802.

31. Sutherland AP, Van Belle T, Wurster AL, et al. (2009): Interleukin-21 is required for the development of type 1 diabetes in NOD mice. Diabetes 58: 1144-1155.

32. Spolski R, Kashyap M, Robinson C, et al. (2008): IL-21 signaling is critical for the development of type I diabetes in the NOD mouse. Proc Natl Acad Sci U S A 105: 14028-14033.

33. Sarra M, Cupi ML, Pallone F, Monteleone G (2012): Interleukin-21 in immune and allergic diseases. Inflamm Allergy Drug Targets 11: 313-319.

34. Strengell M, Sareneva T, Foster D, et al. (2002): IL-21 up-regulates the expression of genes associated with innate immunity and Th1 response. J Immunol 169: 3600-3605.

35. Strengell M, Matikainen S, Siren J, et al. (2003): IL-21 in synergy with IL-15 or IL-18 enhances IFN-gamma production in human NK and T cells. J Immunol 170: 5464-5469.

36. Kasaian MT, Whitters MJ, Carter LL, et al. (2002): IL-21 limits NK cell responses and promotes antigen-specific T cell activation: a mediator of the transition from innate to adaptive immunity. Immunity 16: 559-569.

37. Pinchuk IV, Morris KT, Nofchissey RA, et al. (2013): Stromal cells induce Th17 during Helicobacter pylori infection and in the gastric tumor microenvironment. PLoS One 8: e53798. 
38. Iida T, Iwahashi M, Katsuda M, et al. (2011): Tumor-infiltrating CD4+ Th17 cells produce IL-17 in tumor microenvironment and promote tumor progression in human gastric cancer. Oncol Rep 25: 1271-1277.

39. Caruso R, Fina D, Peluso I, et al. (2007): A functional role for interleukin-21 in promoting the synthesis of the T-cell chemoattractant, MIP-3alpha, by gut epithelial cells. Gastroenterology 132: 166-175.

40. King C, Ilic A, Koelsch K, Sarvetnick N. (2004): Homeostatic expansion of $\mathrm{T}$ cells during immune insufficiency generates autoimmunity. Cell 117: 265-277. 